Aus der chirurgischen Abteilung des Israelitischen Krankenhauses in Hamburg. (Oberarzt Prof. Dr. A ls be r g.)

\title{
Zur Kenntnis des Ulcus coli simplex.
}

Von Dr. Alfred Levy, Assistenzarzt der chirurgischen Abteilung.

Beobachtungen und Beschreibungen von Fällen von einfachem, nicht spezif ischem Dickdarmgeschw ür, einer Erkrankung, die dem Ulcus ventriculi simplex entspricht, finden sich in der deutschen Literatur nur ganz vereinzelt; dia Gesamtzahl der in der deutschen und ausländischen Literatur veröffentlichten Fälle mag sich auf einige Dreißig beziffern. Es ist daher berechtigt im AnschluB an einen in unserem Krankenhause operierten und geheilten Fall über die Erkrankung, ihre Symptomatologie und Pathologie, zu berichten.

E. F., $22 \mathrm{~J}$., Hausmädchen. Familienanamnese o. B. Eltern leben und sind gesund, keine Tuberkulose, insbesondere keine Lungenleiden in der Familie. Pat. erfreute sich stets bester Gesundheit, nur seit ca. $3 \mathrm{~J}$. an $\mathrm{I}-2$ Std. anhaltenden ,,Magenschmerzen" Ieidend, dic so gering waren, daß Pat. sie kaum beachtete. Im Sept. I9I9 erstmalig in einem Krankenhause behandelt wegen Schmerzen in der $r$. Bauchseite, die plötzlich auftraten, $I_{-2}$ Std. intensiv anhielten, um dann mit Blähungen oder Stuhlgang nach Abführmitteln zu verschwinden. Nach Io T. erfolgte bereits wieder Entiaslassung. Wegen derselben Beschwerden wurde sie im Jan. I920 abermals einige Tage in ein Krankenhaus aufgenommen. Seit dicser Zeit leidet Pat. öfter an Stuhlverstopfung und muß Abführmittel gebrauchen. Drei Tage vor der jetzigen Erkrankung klagte Pat. über dumpfe Schmerzen im ganzen Leib, die nach Abgang von Winden und einmaligem Stuhl stets etwas nachließen, die sich jedoch gestern abend in der $r$. Leibseite verstärkten und lokalisierten und seitdem dauernd vorhanden waren. Kein Erbrechen, Stuhl in der Nacht spontan, hart, unter heftigen Schmerzen. Danach wesentlicher Rückgang der Schmerzen, die nach etwa 3 Std. wieder einsetzten.

Aufnahmebef. 9. IV. 1920: Großes, sehr kräftiges Mädchen in ausgezeichnetem Ernährungszustand; Wangen gerötet, Gesichtsaus- 
druck leidend. T. 38 Grad. P. regelmäßig, kräftig. nicht beschleunigt. Zunge: Feucht, etwas belegt. Herz, Lungen $0 . \mathrm{B}$.

Abdomen: Weich, in der 1. Hälfte ohne Widerstand und ohne Schmerzen eindrückbar. R. fühlt man, wenig nach innen vom Mc Burney, einen rundlich-länglichen, klein hühnereigroßen, sehr gut rerschicblichen, mäßig harten Tumor, der vornehmlich in der zentralen Partie druckempfindlich ist. Bauchdeckenspannung ist 1 . gar nicht, r. andeutungsweise vorhanden. Gynäkologische Untersuchung: Uterus retroflektiert, keine Adnexschwellungen.

Nach alledem wird am ehesten an eine Appendicitis gedacht, wenngleich der Tastbefund ernstliche $Z$ weifel an der Diagnose auf. kommen läßt. Dieser einwärts vom Mc Burney fühlbare, auffallend verschiebliche Tumor paßt nicht zum Bilde der Appendicitis, bei der wir, wenn überhaupt, so einen am oder lateral vom $\mathrm{Mc}$ Burney gelegenen lumor finden, der nur selten verschieblich ist, am häufigsten vielmehr der Beckenschaufel fest aufsitzt. Jedenfalls wird die Indikation zum operativen Eingriff für gegeben erachtet.

O peration (Prof.A l s berg) in Allgemeinnarkose: Hautschnitt 3 Querfinger oberhalb und parallel dem Poupartschen Band. Nach Eröffnung der Bauchhöhle dringt trüb-seröse Flüssigkeit hervor. Bein Hervorzichen des Cörums fühlt man an der der Valvula ileocoecalis gegenüberliegenden Wand einen etwa $6: 3 \mathrm{~cm}$ großen, längsgestellten, harten, an der Oberfläche glatten, in der Mitte etwas eingezogenen Tumor. Die rote Färbung unterscheidet sich wesentlich von dem Samt-Rot bei Peritonitis, und läßt Gefäßerweitcrungen und -verzweigungen erkennen. Die anfangs gefühlte Einziehung in der Mitte des Tumors erweist sich als etwa zweimarkstückgroßer, hellerer, mit Fibrinbelägen bedeckter Bezirk. Bei bimanueller Palpation rom Lumen des Darmes aus durch Einstülpung der Cöcalwand fühlt man diesem Bezirke entsprechend ein kraterförmiges, länglich ovales scharfrandiges Geschwür, wobei innere und äußere Fingerkuppe nur durch eine dünne Schicht voneinander getrennt sind. Appendix nach hinten geschlagen, nicht krankhaft verändert. Appendektomie. - Die krankhaft veränderte Partie der Cöcalwand wird eingestülpt und durch weitgreifende Serosanähte in 2 Etagen versenkt, so da $\beta$ normale Serosa aneinander kommt. Naht der Bauchwunde in 2 Etagen ohne Drainage.

I4. IV. Ungestörter Verlauf, Wohlbefinden. I6. IV. Wunde p. p. geheilt. Stuhl erfolgt nur auf Einlauf. Wohlbefinden. - Im histologischen Präparat der Appendix (Serienschnitte) keine pathologischen Veränderungen, insbesondere keine Tbc. 25. IV. Pat. steht auf; Stuhl nur auf Einlauf; wiederholte Stuhluntersuchung auf Tbc. negativ (Hygienisches Institut). II. V. Stuhl erfolgt auf $A$ bführmittel ohne Beschwerden dabei. Körpergewicht I29 Pfund. Geheilt entlassen. 
Im August kam Pat. abermals zur Aufnahme wegen Unterleibsbeschwerden. Es handelte sich um eine Retroflexio uteri mit Menstruationsstörungen. Verdauungsbeschwerden hat Pat. nicht mehr, nur klagt sic zeitweise über Schmerzen bei Druck auf die Operationsnarbe. Sie wurde mit einem Körpergewicht von 134 Pfund aufgenommen und wog bei der Entlassung I 40 Pfund.

$\mathrm{DaB}$ es sich in unserem Falle um ein e in $\mathrm{f}$ a $\mathrm{ch}$ e s Geschwür handelte und $\mathrm{da} B$ wir nicht irgendeine spezifische mit Geschwürsbildung einhergehende Erkrankung des Darmes vor uns hatten, von denen vor allem die Tuberkulose auszuschließen war, ergibt sich daraus, daß keinerlei Anzeichen für Tuberkulose in der Familie der Patientin zu erheben waren; dazu war sie selbst von blühender Gesundheit und bot keinerlei Anhalt für eine tuberkulöse Erkrankung irgendwelcher Organe, zumal die Darmtuberkulose meist sekundärer Natur ist. Ferner treten die tuberkulösen Ulcera des Dickdarms fast stets in der Mehrzahl auf und machen einen Schleimhautkatarrh, der sich durch hartnäckige Diarrhöen äußert, während im vorliegenden Falle nur ein einziges Geschwür vorhanden war, sich bei der Operation die absolut normale Beschaffenheit des Cöcums erwies und die Patientin unter Verstopfung zu leiden hatte. An den entsprechenden Stellen der Serosa sind bei der Tuberkulose des Darmes meist mit bloßem Auge miliare Tuberkel zu erkennen, was intra operationem vermiBt wurde. Während in unserem Falle das Geschwür längsovale Form hatte, werden die tuberkulösen als gürtelförmige bezeichnet, $d$. h. sie stehen mit ihrer Längsrichtung senkrecht zu der des Darmes. Dazu waren im Stuhle der Patientin nie Tuberkelbazillen nachweisbar. Von besonderer Bedeutung für die Berechtigung zur Ablehnung der Tuberkulose dürfte der negative Ausfall der Ponndorfimpfung sein, sowie die bis jetzt andauernde Heilung durch einfache Übernähung.

Ein typhöses oder dysenterisches Geschwür kam nicht in Betracht, ebensowenig Lues; auch Aktinomykose konnte ausgeschlossen werden. An der Berechtigung der Diagnose Ulcus coli simplex kann nicht mehr gezweifelt werden.

Seit der ersten Aufstellung dieses Krankheitsbegriffs durch Cruvelhier dienten die bis heute über die Erkrankung erschienenen Arbeiten nur dem Zwecke kasuistischer Beiträge und bewiesen dadurch zunächst überhaupt das Vorkommen der Er- 
krankung. Jedenfalls findet sich in der deutschen Literatur keine eingehende Würdigung der Erkrankung. Die erste und bis jetzt auch einzige Arbeit, die näher auf die Symptomatologie, Pathologie und Therapie eingeht, befindet sich in der französischen Literatur von $Q$ u én u und $D$ uva ${ }^{1}$ ) die an Hand einer Eigenbeobachtung und von 29 aus der gesamten Literatur gesammelten Fällen der Erkrankung eine eingehende Würdigung zuteil werden lassen. Auch später sind in der Literatur immer nur einzelne Fälle beschrieben worden, so je einer von Zickler, Gobiet, H.M. L y le, Eun i cke und I) u b s, so daß mit unserem Fall die Zahl der bis jetzt veröffentlichten Erkrankungen von ein $\mathrm{f}$ chem Dickdarmgeschwür 36 betragen dürfte. Von diesen wurden 9 geheilt, alle übrigen nahmen einen tödlichen Verlauf, was eine Mortalität von 75 Proz, bedeutet, und zwar erfolgte der Exitus meist infolge von Perforationsperitonitis, seltener (in 3 Fällen) unter Ileuserscheinungen. Von den 36 Erkrankungen entficlen 25 auf Männer, die übrigen I I Kranken waren weiblichen Geschlechts. Auf die verschiedenen Altersstufen verteilten sie sich folgendermaßen:

Unter 20 Jahren 3 Kranke; im Alter

\begin{tabular}{|c|c|c|c|c|c|}
\hline$\because$ & $3 I$ & $\because 40$ & ," & 4 & $\therefore$ \\
\hline$\because$ & $4 \mathrm{I}$ & $\because \quad 50$ & $\because$ & I 2 & $\because$ \\
\hline$\because$ & 51 & $\therefore 60$ & ". & 4 & $\because$ \\
\hline & & $\begin{array}{l}\because 70 \\
\text { Jahre }\end{array}$ & : & 5 & $\because$ \\
\hline
\end{tabular}

Die Lokalisation der IIlcera verteilte sich auf die verschicdenen Abschnitte des Dickdarms wie folgt:

\begin{tabular}{|c|c|c|}
\hline Cöcum & & Fälle \\
\hline Colon ascendens & 8 & $\because$ \\
\hline Flexura hepatica & 3 & , \\
\hline Colon transversum & I & , \\
\hline Flexura lienalis & 3 & " \\
\hline Colon descendens & 4 & \\
\hline Colon pelvinum & 7 & \\
\hline Rektum & 2 & \\
\hline
\end{tabular}

so daß wir das L'Icus also im ganzen Verlaufe des 1)ickdarms antreffen, am häufigsten im Cöcum und Colon ascendens; im Colon transversum fand es sich bisher nur I mal. Die 8 in

I) Quénu und Duval. L'Ulcere simple du Gros Intestin. Revue de Chirurgie 1902. 
Colon ascendens lokalisierten Ulcera saßen meist sehr nahe dem Cöcum, höchstens bis zwei Handbreit über der Einmündung der Appendix. In 3 Fällen fand sich bei der Sektion außerdem noch ein Ulcus ventriculi, in einem Falle noch ein Duodenalulcus ${ }^{1}$ ).

In der makroskopischen Beschreibung der Ulcera stimmen die einzelnen Beobachter ziemlich überein: die Ausdehnung schwankt zwischen Linsen- und Fünfmarkstückgröße, wobei es jedoch nicht unbedingt kreisrunde Form haben muß; oft ist es, wie in unserem Falle, längs-oval, steht alsdann aber mit seinem größten Durchmesser parallel zur Längsrichtung des Darmes; immer ist es trichterförmig, mit der Basis zur Mucosa sehend. Seine Ränder sind scharf, nicht erhaben, der Geschwürsgrund ist von verschiedener Färbung. Bei frischen Ulcerationen ist zunächst nur die Schleimhaut und Submucosa befallen, so daß der Geschwürsgrund von der Muscularis gebildet wird. Bei fortschreitender Entwicklung ist nur noch die Serosa erhalten, wird auch diese befallen, so kommt es zur häufigsten und gefürchtetsten Komplikation der Peritonitis. Die Darmwand in der nächsten Umgebung ist manchmal gar nicht verändert, vielfach aber findet sich, wie im eigenen Fall, eine chronische Entzündung von verschiedener Ausdehnung, die die Darmwand verdickt und knorpelhart erscheinen läßt, so daß in einzelnen Fällen das Darmlumen dadurch kaum mehr für den kleinen Finger durchgängig blieb.

Die bisherigen histologischen Untersuchungen der erkrankten Darmpartie ergaben das Fehlen des Epithels, der Mucosa bzw. noch Submucosa und Muscularis. Die darunterliegenden, noch erhaltenen Schichten haben ebenfalls die normale Struktur eingebüßt und sind einem zellarmen, narbigen Bindegewebe gewichen, das jedoch sehr gefäßreich ist. Auf Serienschnitten fand man ( $Q$ uén u und $D u v a l$ ), daß eine der größeren Arterien durch einen organisierten, wieder mit kleinen Gefäßen verschenen Thrombus völlig verlegt ist. Die übrigen Gefäße der gesunden Darmpartie sind intakt, so daß es sich im Bereiche des Ulcus um eine thrombosierende (nicht obliterierende) Endarteriitis handelt. In ihr haben wir zunächst den hauptsäch-

I) Chvostek, Allgemeine Wiener med. Zeitung 1878 . 
lichsten ätiologischen Faktor zu suchen; wenn es dann durch Koprostase, harte Kotballen, die an der geschädigten Stelle reizend wirken, oder Fremdkörper, oder starke Peristaltik, oder Verdauungsstörungen $\mathrm{u}$. a. in dem in seiner Vitalität geschädigten Bezirk zu einem Epitheldefekt kommt, der, eben infolge der Ernährungsstörung, keine Heilungstendenz zeigt, so haben wir es dann nur noch mit auslösenden Momenten zu tun; jedenfalls darf man die in der Anamnese oft vorhandene habituelle Stuhlverstopfung nach dem geschilderten histologischen Bilde nicht mehr als ätiologischen Faktor ansprechen.

Welches sind nun wieder die Ursachen für jene thrombosierende Endarteriitis? Z i ckler meint in seinem Falle einer uberstandenen Parametritis die Schuld beimessen zu können, die zu einer Thrombose der Gefäße des kleinen Beckens führte, welche wieder zur Thrombosierung eines den betreffenden Darmabschnitt versorgenden Gefäßes Veranlassung gab. Mag die Erklärung auch für einzelne Fälle zutreffen, ausreichend wäre sie in dieser Fassung nur für die I I auf weibliche Personen entfallenden Erkrankungen. Immerhin können ihr gegenüber die übrigen Erklärungsversuche wie Alkoholismus, Berufstoxinämien (Blei, Quecksilber, Arsen), Chlorose, Gefäßwandschädigungen nicht standhalten und man möchte in Anlehnung an Z ickler auch eine überstandene Appendicitis, Cystitis, Epidydimitis für fähig halten, auf entsprechendem Wege und an entsprechender Stelle die Endarteriitis thromboticans hervorzubringen.

Da es bisher nicht gelungen ist, ein Ulcus des Dickdarms vor der Perforation zu diagnostizieren, der richtige Befund sich vielmehr erst bei der Operation oder auf dem Sektionstische ergab, kann über seine Heilungstendenz auch nichts Sicheres ausgesagt werden. Vielleicht sind manche Darmbeschwerden auf ein Ulcus zurückzuführen, das wieder spontan ausheilte; vielleicht kann es auch symptomlos verlaufen und ausheilen, ohne den Befallenen irgendwie belästigt zu haben; sind doch für das Magenulcus schon die Begriffe „Ulcusträger" und „Ulcus$\mathrm{krank} \mathrm{e}$ " unterschieden worden. Bci den bisher bekannten Fällen trat jedoch in weitaus der Mehrzahl (30) eine Perforationsperitonitis auf, d. h. man muß richtiger sagen, es sind fast nur die zur Perforation kommenden Ulcera bekannt geworden, nur die 
mit mangelhafter Heilungstendenz haben durch die schwere Komplikation einer Peritonitis greifbare Symptome gemacht und die „Ulcusträger", die spontan ausheilten, sind eben nicht diagnostiziert worden.

Sind wir nun heute imstande, rückblickend auf Anamnese, Symptome und Verlauf der durch Operation oder Sektion sicher gestellten Fälle von Ulcus coli simplex, einen für die Erkrankung charakteristischen Symptomenkomplex aufzustellen, der für die Folge die Diagnose ermöglicht?

In den einzelnen Krankenberichten findet sich am häufigsten vermerkt: I. Verstopfung, 2. Schmerzen.

Die Verstopfung wird meist als habituelle und sehr hochgradige, oft sogar bis in die frühe Jugend zurückreichende angegeben. Nach Jahren oder nach Jahrzehnten kommt schließ. lich der Schmerz hinzu, dem wir aus verschiedenen Gründen eine diagnostische Bedeutung beimessen dürfen: erstens ist es ein an einer umschriebenen Stelle des Abdomens anfallsweise auftretender Spontan- sowie Druckschmerz, anfänglich von geringer Intensität, mit deren Zunahme die schmerzfreien Zeiten kürzer werden. Schließlich merken die Kranken selbst eine Wechselbeziehung zwischen Verstopfung und Schmerz; crstere, bisher auf bestimmte Abführmittel noch reagierend, wird hartnäckiger, der Schmerz dauert Stunden bis Tage an, bis er unmittel. bar nach einer endlichen abundanten Defäkation spontan schwindet. Diese anfallsweise auftretenden Schmerzen, diese Koliken in Verbindung mit der habituellen Verstopfung, die während der Defäkation ihren Höhepunkt erreichen, um danach sofort zu verschwinden, sind wohl das tinzige Einheitliche und Charakteristische, das man aus den bisherigen Krankenberichten entnehmen kann. Wir sind uns dabei wohl bewußt, daß auch andere Affektionen des Darmes ein ähnliches klinisches Bild geben können, daß lokalisierter Spontan- sowie Druckschmerz, der sich beim Stuhlgang steigert und danach verschwindet, auch nicht selten bei abklingender Appendicitis beobachtet wird.

Darmblutungen treten selten auf; auch bei unserer Kranken, die 3 Monate vor ihrer Erkrankung wegen Darmstörungen auf der inneren Abteilung unseres Krankenhauses behandelt wurde, konnte niemals Blut im Stuhlgang festgestellt werden. Dagegen 
sind bei den beiden Fällen von Ulcus des Rectums reichliche Blutungen aufgetreten.

Erbrechen (vor der Perforation) findet sich nur in einem einzigen Falle angegeben.

Nicht unwichtige Fingerzeige kann auch der Palpationsbefund geben. An der Stelle des lokalen Druckschmerzes findet sich, wie im eigenen Falle sehr deutlich und, wie vereinzelt auch von anderen Autoren angegeben wird, ein mehr oder weniger harter Tumor, je nach Ausdehnung des Ulcus von verschiedener Größe, der durch die Verdickung der Darmwandung in der nächsten Umgebung des Geschwürs zustande kommt.

Die häufigste und gefürchtetste weil zum Tode führende Komplikation ist die Perforation, die in den 36 bekannten Fällen 30 mal aufgetreten ist und die, wie stets, wenn ein Hohlorgan (Magen, Appendix, Gallenblase) perforiert, mit einer Steigerung der Symptome einhergeht. Die konsekutive Peritonitis unterscheidet sich nicht im geringsten von allen anderen Perforationsperitonitiden, sie kann diffus sein oder sich abkapseln und zu einem Bauchabszeß führen.

Andererseits kann das Ulcus auch die Ursache für einen spastischen oder paralytischen lleus abgeben.

Wenn wir, wie oben besprochen, annehmen müssen, da 3 das Ulcus coli simplex häufiger vorkommt als bekannt ist, wenn eben manches Ulcus spontan ausheilt, wenn es "Ulcusträger" gibt, so sind dieses die prognostisch gut verlaufenden Fälle. Bedenkt man aber die Zukunft der Befallenen, berücksichtigt man, daß sie sich in ständiger Lebensgefahr befinden wegen der zu jeder Zeit möglichen Perforation, so müssen wir sagen, daß wir es in jedem Falle mit einer sehr ernsten Erkrankung zu tun haben. Unsere oben gestellte Frage über die Möglichkeit einer sicheren Diagnose müssen wir leider verneinen. Die Verstopfung und die später auftretenden lokalisierten Schmerzen sind zwar von einer gewöhnlichen Darmträgheit, die mit diffusen Schmerzen einhergeht, zu unterscheiden und bieten unter Berücksichtigung der Koliken und Sensationen bei der Defäkation etwas Charakteristisches, aber sie ermöglichen keine sichere Diagnose, zumal sie auch, wie schon bemerkt, bei anderen Affektionen des Dickdarms vorkommen können. Eine allogemeinere Kenntnis dieser 
Erkrankung wird jedoch dazu führen, häufiger an sie zu denken und in manchen Fällen wird man sie alsdann mehr als bisher differentialdiagnostisch in Betracht ziehen müssen.

Was nun die Behandlung des Ulcus coli simplex anbelangt, so haben wir zu unterscheiden, in welchem Stadium der Erkrankung die Patienten in unsere Behandlung kommen, ob bereits eine Perforationsperitonitis vorliegt, oder ob wir noch ror Eintritt derselben eingreifen können, sei es, daß die richtige Dia. gnose gestellt ist, was bisher noch niemals der Fall war, sei es, daß unter einer anderen Diagnose die Laparotomic gemacht werden muß und sich das Ulcus intra operationem zeigt, sei es, da $\beta$ die Schmerzattacken, zusammen mit dem Palpationsbefund die Probelaparotomie indiziert sein lassen. Dic Behandlung der Perforationsperitonitis geschieht nach den bekannten chirurgischen Grundsätzen.

Hat man aus einem der oben angeführten Gründe die Laparotomie gemacht, ohne daß eine Peritonitis besteht, haben die Symptome die Indikation zum Eingriff abgegeben, hat man vielleicht den Verdacht eines Ulcus des Dickdarms, so fragt es sich, ob die Veränderungen der Darmwand äußerlich derart sind, daß man die erkrankte Partie finden kann. Wir sagten schon, daß es bei einem Ulcus zu einer Verdickung und flammenden Rötung in der Umgebung desselben kommt; war vorher durch die Bauch. decken ein Tumor zu fühlen, so wird man einen Fingerzeig für die Lokalisation haben. Beides war bei unserer Patientin der Fall und wir konnten, da der übrige Dickdarm und das Cöcum sonst völlig gesund waren, intra operationem die richtige Diagnose stellen. Ist man so weit, so fragt es sich, welche therapeutischen Maßnahmen zu ergreifen sind. Empfohlen werden:

I. Die Exzision des Geschwürs mit folgender Vernähung;

2. die Resektion der llcocökalgegend, besonders von $\mathrm{Q}$ u én u und $\mathrm{Duval}$ angeraten, als sicherster Eingriff um eine radikale Heilung zu erzielen;

3. erwähnen $Q u e ́ n u$ und $D u v a l$ die Einstülpung der erkrankten Partie;

4. wurde ein Llcus simplex des Rektums vom Anus aus mit dem Thermokauter verschorft und zur Heilung gebracht.

In unserem Falle wurde, entsprechend dem Verfahren bei 
Ulcus ventriculi, dic erkrankte Darmpartie eingestülpt und die Serosa durch 2 Reihen Nähte aneinander gebracht. Die Patientin erfreut sich heute, 8 Monate nach der Operation, einer einwandfreien Gesundheit, insbesondere hat sie keine Leibschmerzen oder Verdauungsstïrungen, hat sogar inzwischen geheiratet, so daß-wir wohl mit Recht die Patientin als geheilt ansehen dürfen. Es ist dieses der erste Fall von Ulcus coli simplex, der durch einfache Einstülpung und Übernähung des Darmes behandelt wurde; der Eingriff darf wohl, auch mit Rücksicht auf das gute Resultat, als die Methode der Wahl gelten, zumal er kein großer ist und von allen angegebenen Methoden die wenigst eingreifende darstellt. 